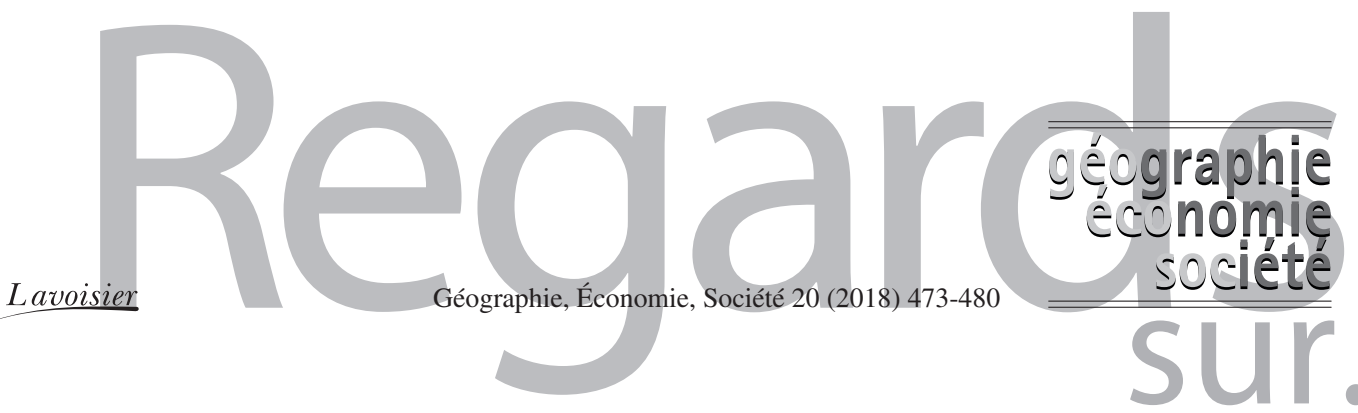

GES participe de manière classique à la vie scientifique par la diffusion des travaux des chercheurs, les comptes rendus de livres et de colloques etc. Nous proposons à travers cette rubrique "Regards sur les questions d'actualité » d'ouvrir la revue aux débats contemporains autour de questions d'actualités qui relèvent de la sociologie, de la géographie, de l'aménagement et de l'économie... L'objectif est de retracer, à partir d'interviews, le parcours de chercheurs et de penseurs provenant d'horizons disciplinaires divers et de recueillir leurs regards sur les grands enjeux spatiaux et sociétaux.

Lise Bourdeau-Lepage $^{*}$ et Leïla Kebir ${ }^{* *}$

\title{
Une interview de Roberta Capello
}

\section{Par Lise Bourdeau-Lepage et Leïla Kebir}

Engagée, passionnée, méthodique et rigoureuse, Roberta Capello s'emploie à montrer et démontrer que les territoires ont leurs spécificités et qu'il est grand temps de prendre cela en compte dans les politiques de développement notamment régionales. Elle nous livre ici son parcours de chercheure qui, discrètement mais sûrement, ouvre des voies pour les femmes dans sa discipline. 


\section{Roberta Capello}

Fonction actuelle : Professeure, Politecnico di Milano
Discipline : Économie, Science régionale
Lieux de vie passés et actuels : Principalement Milan
Thèmes actuels de recherche : Développement économique

régional, innovation, politique de cohésion européenne

Roberta Capello est née en 1962 à Milan. Convaincue par son père que des études d'économie permettraient plus tard de faire beaucoup de choses et séduite par la dimension concrète et proche des réalités de la discipline, elle se lance en 1981 dans des études d'économie générale puis d'économie politique à l'Université Bocconi de Milan. En dernière année de licence, elle suit le cours d'économie régionale de Riccardo Cappellin et découvre alors avec passion les problématiques relatives à l'espace. Elle effectue son mémoire de fin d'études avec Roberto Camagni en 1986, sur le thème de l'innovation et de sa diffusion spatiale. Son diplôme en poche, et souhaitant faire de la recherche, elle rejoint son équipe en tant que chercheure. De 1988-1989, elle effectue un séjour de recherche au Royaume-Uni, au Center for Urban and Regional Development Studies à l'Université de Newcastle upon Tyne. Elle y poursuit ses travaux sur les technologies de l'information, et leur diffusion. De retour à Milan, elle rejoint l'Université Bocconi en tant que chercheure. Elle rencontre Peter Nijkamp et décide de se lancer dans une thèse que ce dernier dirigera à distance. En 1992, elle obtient un poste de chercheure au Politecnico di Milano et soutient l'année suivante sa thèse intitulée "Regional Economic Analysis of Telecommunication Network Externalities » à l'Université libre d'Amsterdam. En 2001, après un passage de trois ans à l'Université de Molise où elle sera professeure associée, elle retourne au Politecnico di Milano et devient professeur ordinaire en 2003. Il y poursuit depuis ses activités. Active auprès des institutions publiques, nationales et européennes, elle coordonne de nombreux projets de recherche. Elle développe avec Roberto Camagni des travaux sur la ville durable, l'impact territorial des politiques régionales, l'innovation et la circulation des connaissances et leur impact sur la croissance des villes et des régions, etc. Auteure foisonnante, Roberta Capello, publie de nombreux articles et ouvrages. Elle publie d'abord en italien (2004) puis en anglais (2007) et en chinois (2015) le manuel de référence Regional economics. Parallèlement à ses recherches, elle s'engage dans la communauté des chercheurs en science régionale. En 1995 elle devient secrétaire de la section italienne de la Regional science association international, (AISRE). Au terme de son mandat, elle rejoint le bureau de l'association européenne, la European regional science association (ERSA) en tant que trésorière. En 2009, elle est la première femme (et seule à ce jour) Présidente de la Regional Science Association International (RSAI). Elle participera dans ce cadre à développer les activités en Amérique du Sud, en créant la section Colombienne tout en préparant le terrain pour la création de la section 
argentine. Roberta Capello reçoit la Laurea Honoris Causa en 2012 de l'Université de Timisoara en Roumanie et, en 2017, le prix de la European Regional Science Association (ERSA) pour l'ensemble de sa carrière. Elle vient de publier Regional Competitiveness, Territory and the City: The Research Programme of an Impressive Mind.

\section{Vous avez mis du temps à vous décider pour vous lancer dans un travail de thèse de doctorat. Cette dernière a porté sur les nouvelles technologies de l'information (qui émergeaient à l'époque) et leur impact sur le développement régional. Comment expliquez-vous cela? Quels sont les apports essentiels de ce travail?}

Au départ, je ne souhaitais pas faire de carrière académique. Je voulais être chercheure et je pensais pouvoir rester à l'université sans faire de thèse de doctorat. Puis, j'ai compris que c'était un peu bizarre de rester dans un tel environnement sans vraiment faire carrière. J'ai alors entrepris une thèse sous la direction de Peter Nijkamp en économie. On était au début des années 1990. Au moment où les technologies de l'information, les réseaux télématiques commençaient à se développer. La question était alors de savoir si, et comment, ces nouvelles technologies allaient permettre le développement des régions. Allaient-elles aider à la convergence entre les régions et à la réduction des disparités de développement? Jusque-là les recherches avaient surtout été théoriques. Dans ma thèse, j'ai développé un modèle de compétition oligopolistique à la Cournot, en montrant que l'adoption de ces nouvelles technologies et en particulier la mise en réseau de deux entreprises pouvaient avoir un impact sur l'équilibre entre les entreprises en question. Du point de vue empirique, j'ai regardé la différence qu'il y avait dans l'emploi des nouvelles technologies au Nord et au Sud de l'Italie et l'impact sur le développement que cela pouvait avoir. Ma thèse a montré que ce n'était pas le niveau d'adoption de la technologie qui était important mais plutôt l'utilisation qu'on en faisait. Dans le Nord, les firmes qui l'avaient adoptée, l'avaient fait pour développer de nouvelles niches de marché; elles étaient devenues beaucoup plus compétitives, tandis que dans le Sud, celles qui l'avaient adoptée avaient continué à faire ce qu'elles savaient faire et n'en n'avaient tiré aucun avantage compétitif.

\section{Dans votre parcours vous avez ensuite travaillé sur de nombreux sujets de science régionale.} Quel a été votre démarche dans vos choix de recherche? Y a-t-il des sujets qui vous ont intéressée plus que d'autres?

Avec Roberto Camagni, nous avons toujours cherché des projets qui portaient sur des thèmes qui étaient soit particulièrement intéressants du point de vue théorique, soit marqués par le fait qu'il y avait peu de résultats empiriques ou soit très intéressants du point de vue des retombées politiques. Lorsqu'a émergé la problématique de la durabilité urbaine qui était très en vogue à la fin des années 1990, nous avons pris en compte les travaux qui, en Angleterre, démontraient l'importance de la ville compacte par rapport à la ville étalée. Le débat était très riche mais il y avait peu d'analyses empiriques du phénomène. Nous nous sommes alors attelés à traduire le concept de la durabilité dans le contexte urbain qui par définition n'est pas naturel. Puis, nous avons analysé la durabilité de différentes villes, au regard de leur taille (à partir de quelle taille une ville devient plus ou moins durable?), de leur organisation interne et de leurs fonctions. Les villes ne sont pas toutes les mêmes. Nous avons obtenu des résultats très intéressants. On a montré, à partir 
d'un échantillon de 58 villes italiennes, que les économies d'agglomération décroissent quand la taille de la ville augmente, ce qui rejoignait ce que disait la littérature depuis des années. Mais ce qui est nouveau, c'est que cette recherche a montré que deux variables fondamentales permettent de déterminer la taille optimale d'une ville. Il s'agit des fonctions urbaines et de l'organisation du système spatial urbain. Les résultats démontrent en effet que les avantages urbains croissants sont dus à la présence d'une quantité élevée de fonctions supérieures dans des villes de même taille. De plus, la croissance des fonctions supérieures en haut de la hiérarchie accroît les coûts de la localisation urbaine, mais à des taux décroissants. À cela s'ajoute le fait qu'à grandeur et à fonctions urbaines égales, les avantages augmentent simultanément avec l'élévation du niveau d'intégration en réseau, une fois dépassée une certaine taille critique. Ce résultat prouve ce que la théorie des « réseaux de ville » affirmait depuis des années.

Mais il y a une question récurrente qui nous a toujours occupés : l'innovation. Il y a cinq ans, on nous a demandé d'analyser la dimension territoriale de l'économie de la connaissance. Où est la connaissance et pourquoi ? comment peut-on la développer? la diffuser dans les régions? Au début, j'avais l'impression que tout avait déjà été dit et en y réfléchissant... nous avons compris qu'il y avait encore beaucoup à dire. Par exemple, l'idée selon laquelle l'innovation se situerait à la frontière des connaissances mondiales ne tient pas! Cela revient à dire que les régions périphériques ne peuvent jamais innover. Or l'innovation est un concept relatif. Cela dépend... une région innove lorsqu'elle développe une activité de production ou un processus qu'elle ne faisait pas auparavant. C'est une innovation pour la région, pas pour le monde. Une fois que nous avons compris cela, nous avons compris que les régions innovent de façon différenciée, elles se situent à une échelle de connaissance complètement différente. Considérer uniquement l'innovation comme ce qui fait avancer la frontière des connaissances qu'on a dans le monde exclut toutes les autres possibilités. En Europe, il n'y a alors plus que Munich, Paris, Londres qui innovent. Quelle politique pouvons-nous mener pour les régions périphériques? Nous avons fait des analyses et identifié ce que nous avons appelé les regional innovation patterns, qui sont les différentes configurations dans lesquelles les régions peuvent innover. Une région qui imite de façon créative, si avant elle ne faisait que de l'imitation passive, et bien c'est déjà un progrès. Et si la région n'imite pas, mais commence à développer de l'innovation de manière endogène, c'est un grand pas en avant. Nous avons donc défini théoriquement trois configurations, allant de l'imitation jusqu'à la création de nouvelles connaissances scientifiques.

Puis, nous avons analysé de manière empirique des régions pour voir où elles se situaient. Nous avons pu montrer que les régions qui imitent sont très efficaces par rapport aux régions qui créent de la connaissance scientifique. Nous avons également montré que, comme toujours, la création de nouvelles connaissances scientifiques a des rendements décroissants en termes de croissance régionale. Ainsi si, dans les régions qui imitent, on investit et que l'on met toutes les forces dans la mise en place d'une innovation par le développement de nouvelles connaissances (du type recherche et développement), ces régions vont perdre en efficacité et en croissance régionale, tout simplement parce que les acteurs ne savent pas quoi faire de la recherche et de l'innovation.

Nous pouvons citer beaucoup d'autres cas. Dans la province de Catane, en Sicile, beaucoup de financements publics ont été investis pour créer la Silicon Valley italienne. 
Des facilités ont été mises en place pour favoriser l'implantation d'entreprises pour le développement de la science. Pour les entreprises en question cela pouvait être attractif : il fait beau toute l'année, on y mange et on peut bien y vivre. Mais du point de vue du PIB de la Sicile, cela n'a rien changé ou presque. Cette politique a détruit le mode de pensée des entreprises locales qui avaient de toutes autres expertises et spécialisations industrielles et qui de ce fait ne sont pas arrivées à bénéficier des effets de débordements (spillovers) de la présence de ces nouvelles entreprises. On revient à la vieille histoire des pôles de croissance, où on crée une firme dominante sans lien avec le tissu local avec pour seul résultat que ça ne se passe pas bien. C'est une façon de relire ce problème d'un point de vue moderne. Nous étions très opposés à l'idée de l'agenda de Lisbonne de l'Union européenne selon lequel la politique d'innovation devait se mesurer par le rapport entre les dépenses de recherche-développement $(\mathrm{R} \& \mathrm{D})$ et le produit intérieur brut; et qu'il fallait que ces dernières soient égales à $3 \%$ du PIB. Nous avons démontré que la plupart des pays étaient très loin de ces $3 \%$. Seules la Suède et la Norvège l'ont déjà atteint. Deux pays en Europe. Et au niveau régional, plus de $20 \%$ des régions sont au-dessous de $0,5 \%$ du PIB. Pourquoi devrions-nous tous développer de la science? Il existe bien d'autres façons de faire de l'innovation. Les brevets ou la grande firme ne sont pas les seuls moyens. Le projet a eu un grand succès du point de vue scientifique et au niveau du programme ESPON. Mais pour ce qui est de la Direction Générale de la Politique régionale (DG Regio) et de la mise en œuvre politique, je n'ai pas vu beaucoup de changement. L'idée de smart specialisation qui devrait apporter le déploiement d'une politique d'innovation liée aux caractéristiques locales, a démontré être encore très attachée à l'idée que l'innovation se mesure à l'aide des brevets déposés. J'ai été invitée il y a deux ans à l'ouverture de la European Week of Cities and Regions, la Commissaire Corina Cretu était présente et j'ai alors souligné que j'étais surprise du fait que la smart spécialisation était finalement quelque chose de déjà-vu car toujours liée aux secteurs industriels.

Il faut selon moi, et comme le préconise la littérature, fonder les politiques d'innovation sur les ressources et non les secteurs. Pour innover, il faut partir des ressources stratégiques de la région et les mobiliser de manière plus efficace. Cela n'a rien à voir avec la présence de grandes firmes, ni avec les brevets, ni avec la technologie. Il faut regarder si la région a des ressources naturelles ou culturelles sur lesquelles le tourisme se développe d'une façon efficace. Je cite volontairement le tourisme qui n'est pas un secteur en soi, mais plutôt une filière. Il y a encore beaucoup de travail à faire pour convaincre les politiques, même à Bruxelles.

\section{Vous vous êtes beaucoup engagée dans les associations de science régionale. Vous avez été la première femme (et seule à ce jour) présidente de la Regional Science Association International. Pour quelles raisons vous y êtes-vous engagée?}

Les expériences que j'ai eues dans ces associations de sciences régionales ont été très importantes pour moi et dans ma carrière, et cela pour de nombreuses de raisons. La première est que j'ai été en contact avec beaucoup de collègues et les conférences sont quelque chose de remarquable. Surtout, ces engagements m'ont permis de développer... la diplomatie et, à la fin, une capacité de leadership. Lorsqu'on est dans une position de présidence, on doit être capable de guider l'association vers un objectif. J'ai commencé très jeune, j'avais 33 ans lorsque je suis devenue secrétaire de l'Association italienne de sciences régionales, j'étais vraiment jeune pour ce poste. Je dois admettre que j'ai toujours eu peur de ce type de position. 
Probablement parce qu'on est des femmes, on voit les choses de manière pratique et concrète. Les hommes eux voient la position que ça leur donne! Je suis devenue secrétaire parce que le collègue qui devait le devenir a refusé au dernier moment car cela représentait trop de travail pour lui. Je m'en souviens encore, pendant la conférence ou cela devait se décider, nous n'avions pas de secrétaire. Roberto Camagni est venu me voir et m'a demandé si je voulais l'être. Quand j'ai répondu non, il m'a demandé quel travail je voulais vraiment faire. J'ai alors compris que c'était une opportunité à saisir. Le président de l'époque était un peu macho et me voyait au début comme sa propre secrétaire, car j'étais une femme et très jeune. Il a compris après une année, que ce n'était pas le cas. Je ne cherche pas les positions mais mon caractère fait que lorsque je les ai, alors je m'emploie à les remplir comme il se doit et je me fais respecter. On a réussi à trouver une bonne manière de fonctionner. À 37 ans je suis devenue trésorière de l'Association européenne et le suis restée pendant six ans. J'ai travaillé avec trois présidents différents (Juan Cuadrado Roura, Borjie Johansson et Roberto Camagni). J'ai beaucoup appris. Ils avaient chacun des personnalités complètement différentes de même que les secrétaires. J'ai notamment commencé à apprendre la diplomatie et la manière de conduire les différents comités auxquels on participait. Lorsque mon mandat s'est terminé j'avais 42 ans. En 2007, à l'âge de 47 ans, Peter Nijkamp et Roger Stough, qui était alors le président sortant de la Regional Science Association International, sont venus me voir à Milan pour me demander si je voulais devenir présidente. Je n'ai pas dormi pendant trois nuits ! Je me demandais ce que j'allais bien pouvoir faire, etc. Puis je me suis dit que je ne pouvais pas refuser. Je me suis donc présentée et suis devenue présidente de l'association mondiale. Je dois dire que ça a été une expérience formidable. J'ai appris l'art du leadership et de la construction de consensus. Parce qu'au sein de l'association internationale c'est compliqué! Déjà à l'échelle européenne ce n'est pas simple. Mais au niveau mondial, il y a les membres américains, japonais, indiens, qui ont des mentalités complètement différentes avec chacun leurs préoccupations et leurs points de vue qui sont issus de cultures diverses. On doit alors être capable de faire la médiation entre des positions différentes et notamment trouver une vision stratégique commune de comment développer l'association. À l'époque on cherchait à développer l'association en Amérique du Sud, ce qui m'a amenée à aller en Colombie et en Argentine. J'y ai rencontré des collègues très intéressants avec qui des sections ont pu être créées dans ces pays pendant mon mandat, qui ne durera que deux ans. J'ai toujours pensé dans notre discipline des sciences régionales, qu'on avait une grande chance d'avoir la Regional Science Association International avec son réseau d'associations dans le monde entier. Il ne faut pas perdre cela et s'adapter aux évolutions du monde, l'élargissement de l'Europe, puis la montée de la Chine, le développement de l'Inde, de l'Afrique...

\section{8 est l'année de la politique de cohésion européenne. Est-ce que cela a encore un sens selon vous? Quels sont les leviers d'actions en termes de politiques régionales? À quelle échelle cela doit-il se faire selon vous?}

La politique de cohésion est un vrai enjeu. Il s'agit surtout d'un problème d'efficacité. Elle est très importante. On ne peut pas penser une Europe unie sans cette politique. Malheureusement, la DG Regio est trop occupée à démontrer le bien-fondé de son existence et l'importance de la politique pour justifier le budget dont elle dispose. On peut le comprendre, mais ce n'est pas ce qu'on peut appeler une vision stratégique. La solution n'est pas dans la bureaucratie et toutes les évaluations, ex-ante, ex-post, etc. mais plutôt, et ce n'est 
pas facile, dans l'architecture de la politique. Architecture qui doit être plus efficace. La smart specialisation n'est pas la solution aux problèmes de la politique de cohésion, et je ne suis pas sûre que la DG Regio ait entendu les critiques qui lui ont été adressées. Or c'est un enjeu très important. Et puis il y a l'identité européenne. Nous avons mené une recherche sur ce sujet et avons eu des échanges avec des collègues : avec toutes les limites que peut avoir une analyse empirique, scientifique, nous avons pu démontrer que la politique de cohésion n'est pas liée à l'identité européenne. C'est-à-dire que si l'Union européenne dépense de l'argent à un endroit, cela ne veut pas dire qu'il y aura un effet sur l'identité européenne locale. Nous devons encore beaucoup travailler sur cela. C'est un vrai problème. Au niveau politique, c'est terrible. Il y a dans beaucoup de pays des situations dramatiques de ce point de vue, l'Italie, le Brexit, etc. C'est un problème qui nous touche, qui nous concerne, un peu moins scientifiquement, mais fortement du point de vue de la société.

Vous avez beaucoup fait de prospective notamment avec le modèle MASST, quelle est votre prévision pour les cinq, dix prochaines années? Quels sont les enjeux de croissance?

Nous venons de terminer l'étude d'un nouveau scénario qu'on appelle le reference scénario, c'est-à-dire un scénario dans lequel sont intégrés les changements structurels qui ont été provoqués par la crise. Si celle-ci a entraîné une décroissance dans les domaines de spécialisation manufacturière, nous observons aujourd'hui un retour de ces dernières en Europe, ce qui se traduit notamment par un retour de l'emploi. L'autre changement structurel se manifeste dans les villes. Les villes moyennes ont montré une capacité à se transformer plus vite que les autres... Le problème, est que cela n'est pas le cas dans toute l'Europe et notamment dans les pays de l'Est dont la dynamique de rattrapage est moins rapide qu'avant. Cela induit des problèmes en termes de convergence et de disparité régionale. Ce qui signifie que les disparités régionales vont encore augmenter entre les pays mais aussi à l'intérieur de ces derniers. Les régions plus riches sont capables de réagir plus vite que les régions pauvres. Si les hypothèses du scénario se maintiennent alors on peut prédire un accroissement des disparités régionales.

\section{Bonus}

Quelle est la chose que vous voudriez pouvoir mesurer?

Ce que j'aimerais vraiment pouvoir mesurer c'est la productivité. C'est quelque chose de très compliqué.

\section{Quel est le film qui vous a marqué?}

Je ne suis pas allée au cinéma depuis trois mois! Je ne me souviens plus.

\section{Quels sont les lieux marquant de votre enfance?}

Ma ville : Milan! Tout le monde me dit : «Tu es de Milan »... et les Italiens me disent : «Toi, tu es très milanaise », mais c'est normal : je suis née là !

\section{Quelle est la question qu'on ne vous a pas posée et que vous auriez aimé qu'on vous pose?}

Vous auriez pu me demander si je suis satisfaite de ce que j'ai fait et quel a été le coût de tout cela. 


\section{Sélection d'ouvrages}

2018, Cohesion Policies and the creation of a European identity: The role of territorial identity, Journal of Common Market Studies, vol. 76(3), pp. 489-503.

2018, Regional innovation evolution and economic performance, Regional Studies, avec Lenzi C. (publié en ligne).

2017, Seminal Studies in Regional and Urban Economics, Springer.

2009, Handbook of Regional Growth and Development Theories, Edward Elgar, avec Nijkamp P. (eds).

2009, Modelling European Regional Scenarios: Aggressive versus Defensive Competitive Strategies, Environment and Planning A, vol. 41(2) pp. 481-504, avec Fratesi U.

2009, Spatial Spillovers and Regional Growth, European Planning Studies, Vol. 17(5), pp. 639-658.

2008, Modelling regional scenarios for the enlarged Europe, Springer, avec Camagni R., Fratesi U. et Chizzolini B.

2007, Regional Economics, Routledge, $1^{\text {st }}$ edition.

2007, A Forecasting Territorial Model of Regional Growth: the MASST Model, The Annals of Regional Science, vol. 41(4), pp. 753-787.

2006, Territorial Impact of European Telecommunications Policy, Scienze Regionali, vol. 5(2), pp. 89-110, avec Spairani A.

1998, Sustainable Cities, special issue of the International, Journal of Environment and Pollution, vol. 10(1), pp. 28-46, avec Nijkamp P.

1994, Spatial Economic Analysis of Telecommunications Network Externalities, Avebury. 